\title{
Fatores de controle e progressão da sepse na Unidade de Terapia Intensiva: uma
}

\section{revisão de literatura}

\author{
Control factors and progression of sepsis in the Intensive Care Unit: a literature review \\ Factores de control y progresión de la sepsis en la Unidad de Cuidados Intensivos: revisión de la
}

literatura

Recebido: 13/10/2021 | Revisado: 20/10/2021 | Aceito: 21/10/2021 | Publicado: 24/10/2021

Cassiane da Silva Portela Pinto

ORCID: https://orcid.org/0000-0002-5732-8555 Universidade do Estado do Pará, Brasil E-mail: cassiane_portela@yahoo.com

Marcello Vieira dos Santos

ORCID: https://orcid.org/0000-0002-3356-1619 Centro Universitário do Estado do Pará, Brasil E-mail: marcellosantos277@gmail.com

Alzinei Simor

ORCID: https://orcid.org/0000-0002-3848-5467 Universidade do Estado do Pará, Brasil E-mail: alzineisimor.enfo@gmail.com

\begin{abstract}
Resumo
Sepse é a presença de degeneração em alguns órgãos ou sistemas com risco de disfunção orgânica que pode ter origem fúngica, viral, bacteriana ou protozoária. Dessa forma, a sepse pode evoluir rapidamente quando diagnosticado de forma tardia e na ausência de uma terapêutica adequada, sendo responsável por $80 \%$ das mortes nas Unidades de Terapias Intensivas (UTI). Ademais, a equipe multiprofissional deve ter como meta a eliminação da infeção, administração terapêutica e controle das funções orgânicas. Trata-se de uma revisão sistêmica da literatura cujo objetivo é descrever os achados acerca dos fatores de controle e da progressão da sepse na Unidade de Terapia Intensiva. Não obstante, foram constatados que pacientes com sepse podem possuir disfunções psicomotoras, pulmonares e renais. Com isso, faz-se necessária uma equipe multiprofissional para a realização do diagnóstico precoce da doença. Por outro lado, o hemograma se mostrou a principal ferramenta para a identificação de sepse. Evidenciou-se que os surgimentos das complicações estão associados com o tempo de internação, uso prolongado de medicamentos e dispositivos invasivos. Não obstante, no perfil epidemiológico dos pacientes, observa-se a prevalência de indivíduos do sexo masculino, com comorbidades pré-existentes, com faixa etária de 18 a 25 anos e principalmente neonatos que nasceram abaixo do peso, apresentam baixa quantidade de vitamina D3 ou apresentar um pré-natal de alto risco. Paralelamente, a realização de um pré-natal seguro e de qualidade se mostrou promissora na prevenção da sepse. Nesse ínterim, é importante que o profissional de saúde tenha compromisso nas técnicas e assistência realizada.
\end{abstract}

Palavras-chave: Unidade de Terapia Intensiva; Diagnóstico precoce; Sepse.

\begin{abstract}
Sepsis is the presence of degeneration in some organs or systems at risk of organic dysfunction that may have fungal, viral, bacterial or protozoal origin. Thus, sepsis can evolve quickly when diagnosed late and in the absence of an adequate assessment, accounting for $80 \%$ of deaths in the Intensive Care Units (ICU). Furthermore, a multiprofessional team must aim at eliminating the infection, administering therapy and controlling organic functions. This is a systemic literature review whose objective is to describe the findings regarding control factors and the progression of sepsis in the Intensive Care Unit. Nevertheless, it was found that patients with sepsis may have psychomotor, pulmonary and renal dysfunctions. Thus, a multidisciplinary team is needed to carry out an early diagnosis of the disease. On the other hand, the blood count presents a main tool for identifying sepsis. It was evident that the appearance of complications is associated with the length of hospital stay, prolonged use of drugs and invasive devices. Nevertheless, in the epidemiological profile of patients, there is a prevalence of males, with preexisting comorbidities, aged between 18 and 25 years and especially neonates who were born underweight, have a low amount of vitamin D3 or have a high-risk prenatal care. At the same time, the realization of a safe and quality prenatal care proved to be promising in the prevention of sepsis. In the meantime, it is important that the health professional is committed to the techniques and assistance provided.
\end{abstract}

Keywords: Intensive Care Unit; Early diagnosis; Sepsis. 


\begin{abstract}
Resumen
La sepsis es la presencia de degeneración en algunos órganos o sistemas con riesgo de disfunción orgánica que puede tener un origen fúngico, viral, bacteriano o protozoario. Así, la sepsis puede evolucionar rápidamente cuando se diagnostica tardíamente y en ausencia de una terapia adecuada, representando el $80 \%$ de las muertes en Unidades de Cuidados Intensivos (UCI). Además, el equipo multidisciplinar debe tener como objetivo eliminar la infección, administrar terapia y controlar las funciones orgánicas. Se trata de una revisión de la literatura sistémica cuyo objetivo es describir los hallazgos sobre los factores de control y la progresión de la sepsis en la Unidad de Cuidados Intensivos. Sin embargo, se encontró que los pacientes con sepsis pueden tener disfunciones psicomotoras, pulmonares y renales. Por tanto, se necesita un equipo multidisciplinar para realizar un diagnóstico precoz de la enfermedad. Por otro lado, el hemograma resultó ser la principal herramienta para identificar la sepsis. Se evidenció que la aparición de complicaciones se asocia con la duración de la estancia hospitalaria, el uso prolongado de fármacos y dispositivos invasivos. Sin embargo, en el perfil epidemiológico de los pacientes, existe una prevalencia de individuos del sexo masculino, con comorbilidades preexistentes, con edades comprendidas entre los 18 y los 25 años, y especialmente los recién nacidos que nacieron con bajo peso, bajos niveles de vitamina D3 o prenatales de alto riesgo. cuidado. Al mismo tiempo, la atención prenatal segura y de calidad se ha mostrado prometedora para prevenir la sepsis. Mientras tanto, es importante que el profesional de la salud se comprometa con las técnicas y la asistencia que se brindan.
\end{abstract}

Palabras clave: Unidad de Cuidados Intensivos; Diagnostico temprano; Septicemia.

\title{
1. Introdução
}

Sepse é a presença de degeneração em algum órgão ou sistemas com risco alarmante à vida secundária, denominado de disfunção orgânica, que ocorre devido a evolução da síndrome de uma resposta inflamatória sistêmica (SIRS), podendo ser fúngica, viral, bacteriana ou protozoária (Moura et al., 2017)

Consequentemente, por conta da alta disseminação da infecção, caso não haja sucesso na realização da terapêutica, a sepse pode evoluir rapidamente para sepse grave ou choque séptico, tornando-a uma doença de alta mortalidade. (Sampaio, 2017) Com isso, o reconhecimento da sepse deve ser feito o mais precoce possível e por qualquer membro da equipe multiprofissional da saúde que esteja assistindo estes pacientes. (Cortez, 2017)

Paralelamente, os primeiros sintomas relacionais ao surgimento dessa síndrome inflamatória sistêmica é a presença de febre $\geq 38,4^{\circ} \mathrm{C}$, frequência cardíaca(FC) $>90 \mathrm{bpm}$, frequência respiratória $(\mathrm{FR})>20$ irpm ou Pressão parcial de dióxido de carbono $(\mathrm{PaCO} 2)>32 \mathrm{mmHg}$ e leucócitos > 12.000 cels/mm3 .Por conseguinte, a evolução do quadro de hipoperfusão orgânica, pode-se incluir acidose láctica, hipoximia, hipotensão, oligúria e até alterações repentinas no estado mental e quanto mais tempo for levado para a identificação dessa patologia, maior será a redução da sobrevida do paciente (Sampaio, 2017)

A Unidade de Terapia Intensiva (UTI) é o ambiente hospitalar, dotada de estrutura de alta complexidade destinada para pacientes que necessitam de constante acompanhamento da equipe multiprofissional e que estejam em estado grave ou em risco iminente de morte, como pacientes com sepse (Almeida, 2017)

Á vista disso, a sepse é a principal causa de morte nas UTIs representando cerca de $80 \%$ da taxa de mortalidade (Junior \& Luiz, 2016). No Brasil, estudos realizados demostram que a doença é responsável por 54,5\% da taxa de letalidade no País (Tieppo et al., 2017).

Dessa forma, as UTIs configuram-se como um ambiente facilitador para o desenvolvimento de quadros de infecções como a sepse, em decorrência de fatores intrínsecos aos pacientes - geralmente imunossuprimidos - e extrínsecos como os períodos de internações prolongados, realização de procedimentos invasivos ou até mesmo uso de cateter, sonda e acessos venosos que são porta de entrada para esses micro-organismo (Sá, Souza \& Santos, 2019).

Mediante isso, a equipe multiprofissional deve traçar estratégias de cuidado ao paciente em UTI visando a identificação do quadro sugestivos de infecção, para que assim garanta medidas de controles eficazes para prevenir a sepse (Sá, Souza \& Santos, 2019). Nesse sentido, é de suma importância identificar os fatores de risco presente no ambiente hospitalar que predispõem o surgimento desse tipo de complicação (Zanon et al, 2018).

Não obstante, a abordagem terapêutica ao paciente com sepse deve-se objetivar tratar o paciente de forma sistêmica, 
avaliando todos os seus padrões orgânicos. Além disso, tem-se como meta a eliminação do processo infeccioso, administração da terapêutica medicamentosa adequada e controle de índice glicêmico e nutricional, a fim de recuperar a homeostase do paciente (Pedrosa, Oliveira \& Machado, 2017).

Mediante isso, o objetivo desse trabalho é descrever os achados na literatura acerca dos fatores de controle e da progressão da sepse na Unidade de Terapia Intensiva.

\section{Metodologia}

Trata-se de uma revisão sistêmica da literatura, cuja finalidade é reunir, analisar, sintetizar e comparar informações e investigações de uma determinada pesquisa, com o intuito reflexivo e para o desenvolvimento de novos estudos (Ercole, Melo \& Alcoforado, 2019). Essa metodologia consiste em 6 etapas: inicialmente, foi definida a pergunta norteadora "Como ocorre a contaminação, o diagnóstico e o progressão da sepse na Unidade de Terapia Intensiva?"

O segundo passo foi a catalogação dos estudos nas bases de dados Scientific Electronic Library (Scielo), National Library of Medicine (PUBMED) e Latino Americana e do Caribe em Ciências da Saúde (LILACS) com a utilização dados seguintes descritores: "sepse", "unidade de terapia intensiva" e "diagnóstico precoce". A posteriori, foi padronizado a seleção dos estudos utilizando os seguintes critérios de inclusão: ano de 2016 a 2021 e idiomas em inglês, espanhol ou português.

A terceira etapa foi destinada para a seleção dos artigos que se enquadravam na temática. Já na penúltima etapa, foi realizado a análise e interpretação dos dados selecionados, a qual foi identificado as seguintes vertentes de análise a certa da sepse: mortalidade durante e após o tratamento, cuidados durante o manejo da doença, medidas para diminuir o risco de contaminação, diagnóstico e qualidade de vida após alta hospitalar.

Por fim, na sexta e última etapa foi realizado a junção e a exposição das informações encontradas e que estão expostas na Figura 1. 
Figura 1 - Fluxograma de seleção dos artigos sobre sepse na UTI.

\section{Estudos encontrados nas bases de dados:}

SCIELO ( $n=277)$ : Palavras-chave: sepse AND unidade de terapia intensiva $(n=142)$; sepse AND diagnóstico precoce $(n=46)$; diagnóstico precoce AND unidade de terapia intensiva $(n=39)$.

LILACS: $(n=1.701)$ Palavras-chave: sepse AND unidade de terapia intensiva $(n=439)$; sepse AND diagnóstico precoce $(n=879)$; Diagnóstico Precoce AND unidade de terapia intensiva $(n=383)$.

PUBMED ( $n=15)$ Palavras-chave: sepse AND unidade de terapia intensiva $(n=11)$; sepse AND diagnóstico precoce $(n=2)$; diagnóstico precoce AND unidade de terapia intensiva $(n=2)$.

Ele

Estudos Excluídos: Foi aplicado o critério de inclusão

$$
\begin{aligned}
& \text { SCIELO: }(n=69) \\
& \text { LILACS: }(n=285) \\
& \text { PUBMED: }(n=9)
\end{aligned}
$$$$
\text { TOTAL: }(n=363)
$$

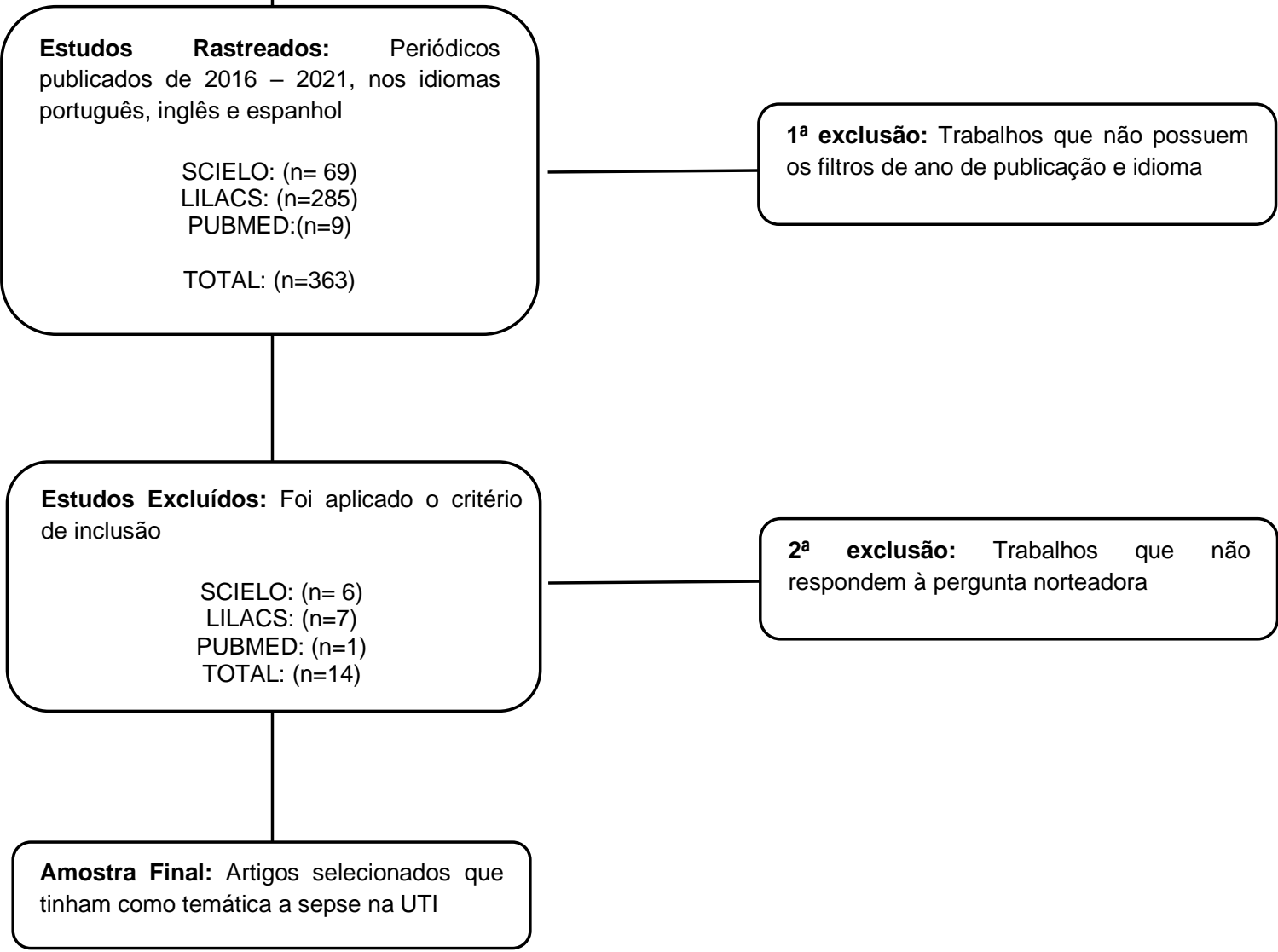

Fonte: Autores.

\section{Resultados e Discussão}

No que diz respeito a catalogação dos artigos revisados, foram encontrados um artigo de 2016, três de 2017, dois publicados em 2018, cinco no ano de 2019 e dois periódicos no ano de 2020 e 2021. Quanto aos países de publicação, a predominância foi de artigos brasileiros sendo encontrado um total de treze documentos, porém foi analisado um artigo da Turquia e um do Uruguai.

Ademais, no delineamento dos trabalhos foram identificados três estudos retrospectivo e observacionais, dois estudos descritivos e estudo de caso controle e um estudo, respectivamente, de prevalência, exploratório, revisão não sistêmica, revisão integrativa da literatura e ensaio clínico, todos esses documentos estão descritos do Quadro 1. 
Quadro 1 - síntese dos artigos encontrados de acordo com título, autores, ano e pais de publicação, delineamento, objetivos e desfecho.

\begin{tabular}{|c|c|c|c|c|c|}
\hline Título & Autores & Ano/ País & Delineamento & Objetivo & Desfecho \\
\hline $\begin{array}{l}\text { Mortalidade oculta em } \\
\text { pacientes sépticos } \\
\text { após a alta da unidade } \\
\text { de terapia intensiva }\end{array}$ & $\begin{array}{ll}\text { RICARDO, I. } & \text { A; } \\
\text { MATEUS, } & \text { H; } \\
\text { PEREIRA, J. G. } & \end{array}$ & $\begin{array}{c}\text { Brasil } \\
2019\end{array}$ & $\begin{array}{c}\text { Ensaio } \\
\text { retrospectivo }\end{array}$ & $\begin{array}{l}\text { Avaliar o impacto da presença de } \\
\text { sepse na mortalidade hospitalar após } \\
\text { alta da unidade de terapia intensiva. }\end{array}$ & $\begin{array}{l}\text { Há um maior risco de } \\
\text { mortalidade para pacientes com } \\
\text { sepse mesmo após a alta } \\
\text { hospitalar. }\end{array}$ \\
\hline $\begin{array}{l}\text { Disponibilidade } \text { de } \\
\text { recursos para o } \\
\text { tratamento da sepse } \\
\text { no Brasil: uma } \\
\text { amostra aleatória de } \\
\text { instituições brasileira }\end{array}$ & 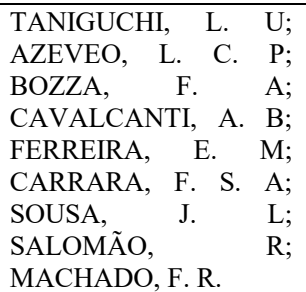 & $\begin{array}{c}\text { Brasil } \\
2019\end{array}$ & $\begin{array}{c}\text { Estudo de } \\
\text { prevalência }\end{array}$ & $\begin{array}{l}\text { Caracterizar a disponibilidade de } \\
\text { recursos a partir de amostra aleatória } \\
\text { representativa das unidades de terapia } \\
\text { intensiva do Brasil. }\end{array}$ & $\begin{array}{l}\text { Um número importante de } \\
\text { unidades não tem condições para } \\
\text { realizar intervenções básicas de } \\
\text { monitoramento e terapêutica em } \\
\text { pacientes sépticos. }\end{array}$ \\
\hline 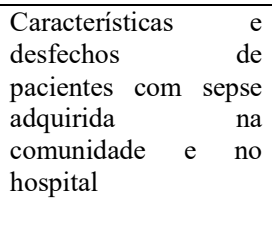 & 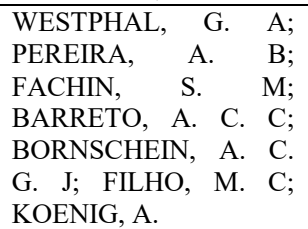 & $\begin{array}{c}\text { Brasil } \\
2019\end{array}$ & $\begin{array}{l}\text { Estudo } \\
\text { retrospectivo de } \\
\text { corte }\end{array}$ & $\begin{array}{l}\text { Comparar as características clínicas e } \\
\text { os desfechos de pacientes com sepse } \\
\text { adquirida na comunidade ou no } \\
\text { hospital. }\end{array}$ & $\begin{array}{l}\text { A sepse adquirida no hospital foi } \\
\text { mais grave e associada com } \\
\text { mortalidade mais alta. }\end{array}$ \\
\hline $\begin{array}{l}\text { Problemas } \\
\text { relacionados a } \\
\text { medicamentos } \\
\text { antimicrobianos em } \\
\text { unidade de terapia } \\
\text { intensiva neonatal }\end{array}$ & $\begin{array}{l}\text { NUNES, B.M; XAVIES, } \\
\text { T. C; MARTINS, R. R }\end{array}$ & $\begin{array}{c}\text { Brasil } \\
2017\end{array}$ & $\begin{array}{l}\text { Estudo } \\
\text { observacional, } \\
\text { prospectivo e } \\
\text { longitudinal }\end{array}$ & $\begin{array}{l}\text { Determinar os principais problemas } \\
\text { relacionados } \\
\text { a medicamentos em neonatos sob uso } \\
\text { de antimicrobianos. }\end{array}$ & $\begin{array}{l}\text { Há o predomínio sobre a } \\
\text { prescrição com subdose de } \\
\text { antimicrobianos, principalmente } \\
\text { aminoglicosídeos. }\end{array}$ \\
\hline $\begin{array}{lr}\text { INCIDÊNCIA } & \text { DE } \\
\text { INFECÇÕES } & \\
\text { PRIMÁRIAS } & \text { NA } \\
\text { CORRENTE } & \\
\text { SANGUÍNEA } & \text { EM } \\
\text { UMA } & \text { UTI } \\
\text { NEONATAL } & \end{array}$ & $\begin{array}{llr}\text { MACHADO, } & \text { C. } & \text { D; } \\
\text { ANTUNES, } & \text { F. } & \text { S; } \\
\text { SOUZA, P. A. } & & \end{array}$ & $\begin{array}{c}\text { Brasil } \\
2017\end{array}$ & $\begin{array}{l}\text { Estudo descritivo } \\
\text { retrospectivo }\end{array}$ & $\begin{array}{l}\text { Identificar } \\
\text { a incidência de Infecções Primárias } \\
\text { da Corrente Sanguínea (IPCS) em } \\
\text { uma UTI neonatal de uma unidade } \\
\text { hospitalar do Estado de Santa } \\
\text { Catarina. }\end{array}$ & $\begin{array}{l}\text { Medidas de educação } \\
\text { permanente diminuem a } \\
\text { incidência das IPCS. }\end{array}$ \\
\hline $\begin{array}{l}\text { Ações do enfermeiro } \\
\text { na identificação } \\
\text { precoce de alterações } \\
\text { sistêmicas causadas } \\
\text { pela sepse grave }\end{array}$ & $\begin{array}{l}\text { GARRIDO, F; TIEPPO, } \\
\text { L; PEREIRA, M. D. S; } \\
\text { FREITAS, R; FREITAS, } \\
\text { W. M; FILIPINI, R; } \\
\text { COELHO, P. G; } \\
\text { FONSECA, F. L. A; } \\
\text { FIORANO, A. M. M. }\end{array}$ & $\begin{array}{c}\text { Brasil } \\
2017\end{array}$ & Estudo descritivo & $\begin{array}{l}\text { Verificar as ações do enfermeiro para } \\
\text { a identificação precoce das alterações } \\
\text { sistêmicas causadas pela sepse } \\
\text { grave relacionadas às alterações } \\
\text { hemodinâmicas, } \\
\text { respiratórias, renais e neurológicas, } \\
\text { dos pacientes } \\
\text { UTIs adulto. }\end{array}$ & $\begin{array}{l}\text { Houve dificuldade por parte dos } \\
\text { enfermeiros na identificação } \\
\text { precoce das } \\
\text { sistêmicas causas pela sepse } \\
\text { grave. }\end{array}$ \\
\hline $\begin{array}{lr}\text { Fatores de riscos } \\
\text { associados } & \text { ao } \\
\text { agravamento da } & \text { sepse } \\
\text { em pacientes em } \\
\text { Unidade de Terapia } \\
\text { Intensiva }\end{array}$ & 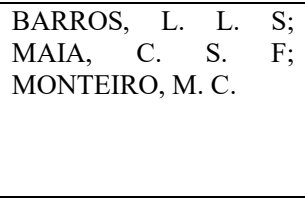 & $\begin{array}{c}\text { Brasil } \\
2016\end{array}$ & $\begin{array}{c}\text { Estudo } \\
\text { observacional } \\
\text { descritivo }\end{array}$ & 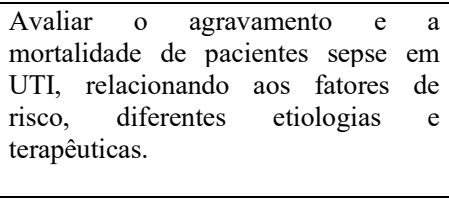 & $\begin{array}{l}\text { Foi identificado uma elevada } \\
\text { taxa de mortalidade por sepse na } \\
\text { UTI, principalmente em } \\
\text { pacientes com choque séptico } \\
\text { com comorbidades. }\end{array}$ \\
\hline $\begin{array}{l}\text { Características } \\
\text { epidemiológicas de } \\
\text { pacientes com sepse } \\
\text { em unidade de terapia } \\
\text { intensiva }\end{array}$ & 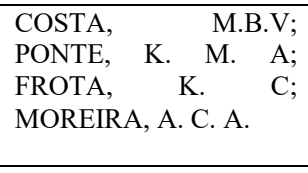 & $\begin{array}{c}\text { Brasil } \\
2019\end{array}$ & $\begin{array}{l}\text { Pesquisa } \\
\text { exploratória- } \\
\text { descritiva }\end{array}$ & $\begin{array}{l}\text { Descrever as características } \\
\text { epidemiológicas de pacientes com } \\
\text { sepse em UTI. }\end{array}$ & $\begin{array}{l}\text { Foram encontradas relações } \\
\text { sobre os dados epidemiológicos } \\
\text { e sua relação com a aplicação de } \\
\text { novos recursos, tecnologias e } \\
\text { tratamentos. }\end{array}$ \\
\hline $\begin{array}{l}\text { The challenges of } \\
\text { neonatal sepsi } \\
\text { mannagement }\end{array}$ & $\begin{array}{l}\text { PROCIANOY, R. S; } \\
\text { SILVEIRA, R. C. }\end{array}$ & $\begin{array}{c}\text { Brasil } \\
2020\end{array}$ & $\begin{array}{l}\text { Revisão não } \\
\text { sistêmica }\end{array}$ & $\begin{array}{l}\text { Apresentar evidências atuais na } \\
\text { etiologia, fatores de risco, diagnóstico } \\
\text { e manejo da sepse neonatal precoce e } \\
\text { tardia. }\end{array}$ & $\begin{array}{l}\text { A observação contínua do } \\
\text { paciente, a valorização dos } \\
\text { sinais clínicos e observar fatores } \\
\text { de riscos são fundamentais para } \\
\text { a suspeita diagnóstica. }\end{array}$ \\
\hline $\begin{array}{lr}\text { Razão } & \text { neutrófilo- } \\
\text { linfócito } & \text { no } \\
\text { diagnóstico } & \text { precoce } \\
\text { de sepse em unidade } \\
\text { de terapia intensiva: } \\
\text { um estudo de caso- } \\
\text { controle }\end{array}$ & 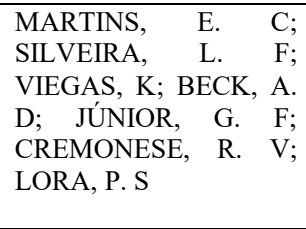 & $\begin{array}{c}\text { Brasil } \\
2019\end{array}$ & $\begin{array}{l}\text { Estudo de caso- } \\
\text { controle }\end{array}$ & $\begin{array}{l}\text { Avaliar a razão neutrófilo- -linfócito } \\
\text { na predição de sepse e morta- lidade } \\
\text { em pacientes admitidos em uma } \\
\text { unidade de terapia intensiva. }\end{array}$ & $\begin{array}{l}\text { A razão neutrófilo-linfó- cito e } \\
\text { os neutrófilos bastonados em } \\
\text { com- binação com outros } \\
\text { parâmetros podem ser } \\
\text { marcadores na detecção precoce } \\
\text { de sepse em terapia intensiva. }\end{array}$ \\
\hline
\end{tabular}




\begin{tabular}{|c|c|c|c|c|c|}
\hline $\begin{array}{l}\text { Um sistema eletrônico } \\
\text { de alerta ajuda a } \\
\text { reduzir o tempo para } \\
\text { diagnóstico de sepse }\end{array}$ & $\begin{array}{lrr}\text { WESTPHAL, } & \text { G. } & \text { A; } \\
\text { PEREIRA, } & \text { A. } & \text { B; } \\
\text { FACHIN, } & \text { S. } & \text { M; } \\
\text { SPEROTTO, } & & \text { G; } \\
\text { GONÇALVES, } & \text { M; } \\
\text { ALBINO, } & \text { L; } \\
\text { BITTENCOURT, } & \text { R; } \\
\text { FRANZINI, V. } & \text { R; } \\
\text { KOENIG, A. } & & \\
\end{array}$ & $\begin{array}{c}\text { Brasil } \\
2018\end{array}$ & $\begin{array}{l}\text { Estudo } \\
\text { observacional de } \\
\text { coorte }\end{array}$ & $\begin{array}{l}\text { Descrever os efeitos de melhorias } \\
\text { sucessivas nos sistemas de alerta } \\
\text { precoce para identificação de pa- } \\
\text { cientes com sepse. }\end{array}$ & $\begin{array}{l}\text { Sistemas eletrônicos auxiliam na } \\
\text { redução do tempo entre a } \\
\text { triagem e o diagnóstico e entre o } \\
\text { diag- nóstico e o início da } \\
\text { antibioticoterapia em pacientes } \\
\text { com sepse. }\end{array}$ \\
\hline $\begin{array}{l}\text { Role of serum }(1,3)- \\
\beta \text {-d-glucan assay in } \\
\text { early diagnosis of } \\
\text { invasive fungal } \\
\text { infections in a } \\
\text { neonatal intensive } \\
\text { care unit is }\end{array}$ & $\begin{array}{lrr}\text { SHABAAN, } & \text { A. } & \text { E; } \\
\text { ELBAZ, L. M; } & \text { EL- } \\
\text { EMSHATY, W. } & \text { M; } \\
\text { SHOUMAN, B. } & \end{array}$ & $\begin{array}{c}\text { Brasil } \\
2018\end{array}$ & $\begin{array}{l}\text { Ensaio clínico } \\
\text { multicêntrico } \\
\text { prospectivo }\end{array}$ & $\begin{array}{l}\text { avaliar o desempenho diagnóstico do } \\
\text { nível de }(1,3)-\beta \text {-D-glucano no soro } \\
\text { para diagnóstico precoce de fungemia } \\
\text { invasiva em neonatos de alto risco. } \\
\text { Estudar o padrão microbiológico das } \\
\text { culturas de sepse neonatal de início } \\
\text { tardio. }\end{array}$ & $\begin{array}{l}(1,3)-\beta \text {-D-glucano possui } \\
\text { sensibilidade limitada com } \\
\text { especificidade e valor preditivo } \\
\text { negativo excelentes que } \\
\text { possibilitam seu uso e ajudam na } \\
\text { exclusão de infec cão fúngica } \\
\text { invasiva neonatal. }\end{array}$ \\
\hline $\begin{array}{l}\text { Infección grave } \begin{array}{r}\text { por } \\
\text { estreptococo }\end{array} \\
\text { grupo B en la Unidad } \\
\text { de } \quad \text { Cuidados } \\
\text { Intensivos Pediátricos } \\
\text { del Centro } \\
\text { Hospitalario Pereira } \\
\text { Rossell entre los años } \\
2007 \text { y } 2017 \\
\end{array}$ & $\begin{array}{ll}\text { RODRÍGUEZ, } & \text { A; } \\
\text { TELECHEA, } & \text { H; } \\
\text { MENCHACA, A. } & \end{array}$ & $\begin{array}{l}\text { Uruguai } \\
2021\end{array}$ & $\begin{array}{l}\text { Estudo descritivo- } \\
\text { retrospectivo }\end{array}$ & $\begin{array}{l}\text { conhecer a incidência de sepse tardia } \\
\text { escrever as características } \\
\text { epidemiológicas e clínicas da sepse } \\
\text { tardia em crianças internadas na UTI. }\end{array}$ & $\begin{array}{l}\text { a sepse tardia está associada a } \\
\text { significativa morbimortalidade } \\
\text { em pediatria. }\end{array}$ \\
\hline $\begin{array}{l}\text { Sepse após a alta da } \\
\text { UTI: um problema de } \\
\text { saúde pública }\end{array}$ & $\begin{array}{l}\text { TEIXEIRA, C; ROSA, } \\
\text { R. G; FRIEDMAN, G. }\end{array}$ & $\begin{array}{c}\text { Brasil } \\
2021\end{array}$ & $\begin{array}{l}\text { Revisão de } \\
\text { literatura }\end{array}$ & $\begin{array}{l}\text { descrever sobre a morbimortalidade a } \\
\text { longo prazo dos pacientes } \\
\text { sobreviventes de sepse. }\end{array}$ & $\begin{array}{l}\text { Asconsequências da sepse vão } \\
\text { além da alta hospitalar, com } \\
\text { diminuição da qualidade de vida, } \\
\text { sequelas e aumento da } \\
\text { mortalidade após a saída da UTI. }\end{array}$ \\
\hline $\begin{array}{l}\text { Asociación entre la } \\
\text { concentración de } \\
\text { vitamina D y la sepsis } \\
\text { neonatal } \\
\text { extrahospitalaria de } \\
\text { aparición tardía. }\end{array}$ & $\begin{array}{l}\text { BILGIN, S. } \\
\text { GONULAL, D. }\end{array}$ & $\begin{array}{c}\text { Turquia } \\
2020\end{array}$ & $\begin{array}{l}\text { Estudo de caso e } \\
\text { controle }\end{array}$ & $\begin{array}{l}\text { determinar a relação entre os níveis de } \\
\text { vitamina } D \text { materno e infantil e sepse } \\
\text { de início tardio. }\end{array}$ & $\begin{array}{l}\text { A concentração de } 25(\mathrm{OH}) \mathrm{D} \\
\text { foi menor em bebês a termo com } \\
\text { sepse de início tardio e suas } \\
\text { mães em comparação com o } \\
\text { grupo controle. }\end{array}$ \\
\hline
\end{tabular}

Fonte: Autores.

O diagnóstico da sepse deve ser feito de forma cautelosa, principalmente de forma precoce em que os sintomas podem estar pouco presentes. Além disso, a doença pode afetar vários órgãos ou sistemas, tornando os sintomas variados e com pouca especificidade (Procianoy \& Silveira, 2019; Cristina et al., 2019)

Diante disso, foi observado a importância de se atentar a todas as alterações nos exames físicos do paciente, principalmente para a presença de hipertermia (temperatura $>38^{\circ}$ ) ou hipotermia (temperatura $<36^{\circ}$ ), FC $>90 \mathrm{BPM}, \mathrm{FR}>20$ RPM e/ou PaCO2> $32 \mathrm{mmHg}$. De forma complementar, o hemograma poderá apresentar leucocitose (>12.000mm3) ou leucopenia $(<4.000 \mathrm{~mm} 3)$, número de bastonetes maior que 10\%, proteína C-reative> 1,5 mg/dl (Martins et al., 2019; Garrido et al., 2017; Rodríguez, Telechea \& Menchaca, 2017).

Além disso, os achados também destacaram a necessidade de avaliar o nível de consciência dos pacientes, presença de convulsão, delírio e agitações psicomotoras podem ser sugestiva do avanço da sepse ou até mesmo de disfunção do sistema nervoso central. Outrossim, também pode-se avaliar funções pulmonares, como alterações do padrão respiratório e secreção traqueal, e renais, com a presença de oligúria (Garrido et al., 2017; Rodríguez, Telechea \& Menchaca, 2017).

Com isso, Westphal et al e Garrido et al abordam a necessidade da equipe multidisciplinar para os achados precoces da doença, além de médicos e enfermeiros, o treinamento dos técnicos de enfermagem pelo próprio hospital também auxiliarem no diagnóstico precoce (Westphal et al., 2018; Garrido et al., 2017).

Além disso, o uso de tecnologias se mostrou promissora para o desenrolar do desfecho clínico, alertar eletrônicos aos telefones dos enfermeiros quando forem achados pacientes de risco através de um algorítmico associado ao Modified Early Warning Score (MEWS), permite encontrar de forma prematura patologias com evolução rápida, como a própria sepse 
(Westphal et al., 2018).

Paralelamente, o diagnóstico precoce pode ser feito de forma mais precisa quando aliado à tecnologia de algoritmos e de comunicação, permitiu a redução da taxa de mortalidade e diminuição do tempo de tratamento aos pacientes de sepse na UTI (Westphal et al., 2018).

De outro modo, segundo Martins et al, o hemograma caracteriza-se por um exame de baixo custo e que possibilita o auxílio para o diagnóstico de várias patologias de forma precoce. Por ser de baixo custo, esse exame possui bastante relevância principalmente em países com o sistema de saúde público deficitário, como o Brasil (Martins et al., 2019; Shabaan et al., 2018).

Dessa maneira, a razão neutrófilo-linfócito (RNL) se mostrou bastante promissora, já que além de não acrescentar custos ao laboratório, apresenta sensibilidade maior que 80\%, apesar de ser pouco específico (CRISTINA et al., 2019). No caso da sepse, o RNL apresentou média de 10,7 n/uL, já em outros casos, a razão era de apenas 6,5 n/uL, o que mostra uma diferença de 4,2 n/uL, uma vez que na sepse, o aumento do neutrófilos é alto se comparado com o de linfócitos (Martins et al., 2019).

Por outro lado, Martins et al também cita a comparação entre o número de neutrófilos bastonados e dos sedimentados para o diagnóstico da sepse, em que os bastonados são superiores aos sedimentarem em pacientes que apresentem infecções generalizadas (Martins et al., 2019).

Ademais, o 1,3-beta-D-glucano (BG) apresentar um importante exame para a identificação de infecções invasivas fúngicas (IFI), principalmente em UTI's neonatais, visto que esse componente está presente na parede celular de alguns fungos e se encontrará elevada já no início da sepse fúngica (Shabaan et al., 2017). Desse modo, o BG apresentou valor superior a $125 \mathrm{pg} / \mathrm{ml}$ nos pacientes com risco de caso de fungemia definida. Por outro lado, valores inferiores a $99 \mathrm{pg} / \mathrm{ml}$ descaram em até 90,7\% a presença de fungemia (Shabaan et al., 2017).

Outro exame que suma importância para a sepse é o Lactato. Foram encontradas literatura com controversa sobre o uso do lactato para o diagnóstico de infeções sistêmica, principalmente devido ao seu baixíssimo grau de especificidade (Teixeira, Rosa \& Friedman, 2021). Entretanto, seu exame é bastante utilizado nas UTI's para avaliar a evolução da doença, sendo que esse marcador tende a diminuir quando a terapia antibiótica estiver fazendo efeito (Teixeira, Rosa \& Friedman, 2021).

Evidenciou-se, com a análise dos artigos que dentre as inúmeras complicações que afetam os pacientes críticos a sepse, o choque séptico e as disfunções orgânicas são as principais e ainda responsáveis pelo aumento das taxas de morbidade e mortalidade nas Unidades de Terapia Intensiva (UTI's). Dessa forma, diversos fatores estão associados ao surgimento dessas complicações como tempo de internação, uso prolongado de medicamentos, dispositivos invasivos, capacitação dos profissionais nesse cuidado e ainda déficit estrutural das instituições (Garrido et al., 2017; Ricardo, Mateus \& Pereira, 2019).

Não obstante, dois artigos traçaram o perfil dos pacientes que foram internados em UTI's em decorrência da sepse, a prevalência era de pacientes do sexo masculino com a faixa etária de 18 a 65 anos com tempo de internação de até 24 dias e as causas das admissões foram principalmente as clínicas (Ricardo, Mateus \& Pereira, 2019; Barros, Maia \& Monteiro, 2016).

Mediante isso, observa-se particularidades epidemiológicas desses pacientes que devem ser levados em consideração no desenvolvimento de ações e estratégias de cuidado, bem como para a identificação precoce da sepse. Ademais, quanto ao período de internação do paciente com sepse é um fator de grande influência no seu prognostico, uma vez que a permanência do paciente na UTI exige maiores números de procedimento e cuidados intensivos podendo por vezes aparecerem outros focos infecciosos em decorrência dessa permanência (Westphal et al., 2019).

Em relação aos focos de infecção mais recorrente, identifica-se o predomínio de infecção e disfunções neurológicas, pulmonares, no trato urinário e no abdômen (Westphal et al., 2019). A vista disso, verifica-se com os trabalhos que os 
principais agente etiológicos da sepse foram as bactérias, tendo destaque a família Enterobacteriaciae, Staphylococcus aureus, Staphylococcus coagulase-negativa, Neisseria meningitidis, micobacteérias e Clostridium tetani (Machado, Antunes \& Souza, 2017; Barros, Maia \& Monteiro, 2016).

Mediante a literatura, muitos pacientes desenvolvem o quadro de sepse após a admissão nas UTI's em decorrência de procedimentos invasivos como cateterismo venoso central, sonda nasogástrica, sonda vesical de demora ou alívio e uso prologado da ventilação mecânica, permitindo que os micro-organismos entrem na microbiota do paciente, agravando o seu quadro infeccioso (Costa et al., 2019; Machado, Antunes \& Souza, 2017).

De outro modo, o desenvolvimento da sepse neonatal é mais delicado, uma vez que, além de não está com o seu sistema imunológico bem desenvolvido, o recém-nascido não consegue expressar de forma clara o que está sentindo, o torna o diagnóstico muito mais delicado (Procianoy \& Silveira, 2019).

Dessa forma, a equipe multidisciplinar do hospital e/ou da UTI neonatal deve levar em consideração o histórico do parto, principalmente quando houver a presença de Streptocococus agalactiae em gestantes que não tenham realizado a profilaxia intraparto, Ruptura de mais de 18 horas da membrana coriônica e/ou presença de corioamnionite pode aumentar a probabilidade de desenvolver sepse no neonato de 4 a 25\% (Procianoy \& Silveira, 2019).

Além disso, foi avaliado a quantidade de Vitamina D3 em neonatos e concentrações inferiores a 15,45 ng/ml estavam mais os associados ao surgimento de sepse de origem tardia nesses recém-nascidos (Bilgin \& Gonulal, 2020)

Concomitantemente, foi observado que neonatos que nasceram abaixo do peso, ou seja, com menos de $1500 \mathrm{~g}$, apresentaram um aumento de $2,4 \%$ a $20,4 \%$ em infecções invasivas fúngicas, principalmente quando associado a Candida albicans, que é a espécie mais associada a sepse fungica nas UTI’s neonatais do Brasil (Shabaan et al., 2017)

Quanto mais tardio for a descoberta e o início do tratamento contra a sepse maior será a taxa de morbimortalidade daquele recém-nascido, chegando a 50\% de mortalidade e dos que sobrevivem, 36\% apresentaram sequelas para a resposta da vida, como disfunção auditiva, visuais e epilepsia secundária (Procianoy \& Silveira, 2019; Rodríguez, Telechea \& Menchaca, 2017).

Ademias, as comorbidades pré-existentes como a Diabetes, Hipertensão e as Neoplasias configuram-se como fatores de risco de destaque para o agravamento da sepse, uma vez que essas patologias acarretam significativas alterações fisiológicas como disfunções no endotélio, distúrbios cardiopulmonares - DPOC, insuficiência cardíaca- e cerebrovasculares, além disso as neoplasias aumento a produção de Óxido Nítrico e consequentemente a diminuição da pressão arterial (Barros, Maia \& Monteiro, 2016).

Outrossim, as internações em decorrência da sepse representam um grande problema de saúde pública não apenas pelos elevados custos hospitalares para tratamento desses pacientes, bem como a falta do aporte estrutural dessa instituição para lidar com esses pacientes. Esse cenário é evidenciado nos estudos, pela falta de laboratórios de microbiologia não proporciona acesso a hemocultura, ausência de bancos de sangue e medicações especificas - vasopressores, cristaloides, antibióticos- falta de estrutura para monitoramento e até mesmo ausência de protocolos nas instituições (Taniguchi et al., 2019).

Em consideração a isso, Garrido et al expõe que os profissionais de saúde enfrentam entraves no cuidado ao paciente com sepse, devido razões institucionais e até a falta da capacitação dos profissionais, estes não tendo o aporte teórico para realizar leitura de exames laboratoriais falta de treinamento para a realização de procedimento de baixo a alto risco.

Perante o exposto, se apresenta como fator de grande importância para a redução das taxas de mortalidade na Unidade de Terapia Intensiva que seja realizado uma assistência integra e de qualidade, que o conjunto de ações desenvolvidas pelos profissionais nesse setor tenha como objetivo principal, o diagnóstico precoce e o tratamento eficaz do paciente uma vez que a eficácia dessa assistência é o que garante o sucesso da reversão do quadro e a promoção da saúde. 
Recorrentemente, os pacientes nas UTI's são tratados com antimicrobianos, principalmente a Cefepima e a Vancomicina. Contudo, o uso dessa terapia medicamentosa se mostra complexa devido a dificuldade na posologia, resistência bacteriana ao medicamento, toxicidade em decorrência da inadequação de doses e frequências de administração que acarretam em disfunções orgânicas e ainda a dificuldade de alguns pacientes na excreção do fármaco (Nunes, Xavier \& Martins, 2017; Costa et., 2019).

Por ter o quadro evolutivo muito rápido, a sepse pode trazer vários prejuízos para o paciente mesmo após a sua recuperação na UTI, principalmente se esse paciente for idoso. Isso ocorre devido a reinfecções e pelo alto poder de disseminação das infecções generalizadas, que podem acabar desenvolvendo processos patológico em órgãos ou agravar alguns problemas já existentes, piorando o quadro de doenças já existentes, como Doença Pulmonar Obstrutiva Crônica, Insuficiência Cardíaca ou renal, pneumonia aspirativa (Teixeira, Rosa \& Friedman, 2021; Ricardo, Mateus \& Pereira, 2019)

A partir disso, Teixeira et al mostra que $40 \%$ dos pacientes que sobreviveram a um quadro de sepse tiverem que ser readmitidos para a internação. Além de que $16 \%$ a $36 \%$ de todos esses sobreviventes vieram a óbito no primeiro ano de após a sua alta (Teixeira, Rosa \& Friedman, 2021).

Além do mais, pacientes que retornaram de um tratamento de sepse na UTI relataram dificuldade para retornar as atividades cotidianas, principalmente quando associada a sequelas na cognitivas permanentes ou na limitação da capacidade física, o que proporciona com diminuição da qualidade de vida dessas pessoas (Teixeira, Rosa \& Friedman, 2021; Ricardo, Mateus \& Pereira, 2019)

\section{Considerações Finais}

No presente trabalho, destacou-se a importância da equipe multiprofissional para o diagnóstico precoce da sepse, uma vez que a sepse é um problema com sintomatologia variada e com baixa especificidade.

Para tanto, é importante o papel da própria instituição de saúde em instrui certos profissionais para saber identificar sintomatologias que possam levar o paciente ao desenvolvimento de um quadro de maior risco, como a sepse, como técnicos de enfermagem, que estão em constante contato com os pacientes, mas que nem sempre possuem esse conhecimento.

De forma análoga, foi observado a necessidade de inclusão de tecnologias que permitam analisar os dados dos exames dos pacientes por meios de algoritmos e relacionar esses achados ao risco de desenvolvimento de complicações nas UTI's, principalmente públicas, além do envio de mensagem de alertar sobre esses pacientes para os enfermeiros responsáveis. Nesse sentido, o uso dessas pode resultar na diminuição da taxa de mortalidade dos pacientes em geral, além de facilitar o diagnóstico mais precocemente de patologias, como o da sepse.

Não obstante, a realização do pré-natal também se mostrou promissora para a prevenção da sepse em UTI's neonatais, visto que os nascidos abaixo do peso ou com baixos níveis de vitamina D3 estão mais susceptíveis a aquisição de infecções generalizadas tardias ou precoces.

Ademais, é primordial ressaltar a importância das unidades de saúde em disponibilizar acompanhamento médico e psicológico a pacientes com sucesso no tratamento e/ou que já receberam alta hospitalar, já que essas pessoas possuem alta taxa de mortalidade mesmo após o fim do tratamento e da diminuição da sua qualidade de vida devido às sequelas que podem ser adquiridas pelo desenvolvimento das infecções generalizadas.

Além do mais, cabe ao poderio público o envio de recursos às instituições para que possam deter de infraestrutura, profissionais, medicamentos para assim garantir uma assistência de qualidade e completa ao paciente não tendo negligenciado o seu direito básico à saúde

Conforme o exposto no trabalho, é de suma importância que os profissionais da saúde ao realizadas procedimentos invasivos tenha total compromisso de que sejam feitas com técnicas assépticas, com intuito de diminuir o risco de infecção 
nesse ambiente hospitalar.

Ademais, essa temática é fundamental para melhoria do processo de trabalho e da saúde dos pacientes. Á vista disso, se justifica a construções de trabalhos científicos sobre sepse, principalmente com relação a taxa de mortalidade alta em pacientes que receberam alta hospitalar após um quadro séptico, bem como suas publicações visando contribuir para formulação de ações assistenciais.

\section{Referências}

Almeida, D. V. D. (2017). Perfil do paciente idoso internado em Unidade de Terapia Intensiva neurológica em um hospital público no Distrito Federal. Dissertação (Mestrado em Enfermagem-Universidade de Brasília, Brasília).

Barros, L. L. S., Maia, C. S. F. \& Monteiro, M. C. (2016). Fatores de risco associados ao agravamento de sepse em pacientes em Unidade de Terapia Intensiva. Cadernos Saúde Coletiva, 24, 388-396.

Bilgin, S. B. \& Gonulal, D. (2020). Asociación entre la concentración de vitamina D y la sepsis neonatal extrahospitalaria de aparición tardía. Arch. argent. pediatr, 265-272.

Cortez, L. M. M. R. (2017). Diagnóstico precoce da sepse no paciente oncológico internado na uti: uma análise de protocolo. Dissertação (Mestrado em Ciências- Fundação Antônio Prudente.

Costa, M. B. M., Ponte, K. M. A., Frota, K. C. \& Moreira, A. C. A. (2019) Características epidemiológicas de pacientes com sepse em unidade terapia intensiva. Revista de epidemiologia e controle de infecção. V. 9, n.4.

Ercole, F. F., Melo, L. S. \& Alcoforado, C. L. G. C. (2014). Revisão integrativa versus revisão sistemática. Revista Mineira de Enfermagem, 18(1), 9-12, 2014.

Garrido, F., Tieppo, L., Pereira, M. D. S., Freitas, R., Freitas, W. M., Filipini, R., Coelho, P. G., Fonseca, F. L. A. \& Fiorano, A. M. M. (2017). Ações do enfermeiro na identificação precoce de alterações sistêmicas causadas pela sepse grave. ABCS Health Sciences, 42(1).

Machado, C. D., Antunes, F. S. \& Souza, P. A. (2017). Incidência de infecções primárias na corrente sanguínea em uma uti neonatal. Arquivos Catarinenses de Medicina, 46(2), 88-96.

Martins, E. C., Silveira, L. F., Viegas, K., Beck, A. D., Júnior, G. F., Cremonese, R. V. \& Lora, P. S. (2019). Razão neutrófilo-linfócito no diagnóstico precoce de sepse em unidade de terapia intensiva: um estudo de caso-controle. Revista Brasileira de Terapia Intensiva, 31, 64-70.

Moura, J. M., Sanches, E., Pereira, R., Frutuoso, I. \& Werneck, A. L. (2017). Diagnóstico de sepse em pacientes após internação em unidade de terapia intensiva. Arquivos de Ciências da Saúde, 24(3), 55-69.

Nunes, B. M., Xavier, T. C. \& Martins, R. R. (2017). Problemas relacionados a medicamentos antimicrobianos em unidade de terapia intensiva neonatal. Revista Brasileira de Terapia Intensiva, 29, 331-336.

Procianoy, R. S. \& Silveira, R. C. (2020). The challenges of neonatal sepsis management. Jornal de pediatria, 96, 80-86.

Ricardo, I. A., Mateus, H. \& Pereira, J. G. (2019). Mortalidade oculta em pacientes sépticos após alta da unidade de terapia intensiva. Revista Brasileira de Terapia Intensiva, 31, 122-128.

Rodríguez, A., Telechea, H. \& Menchaca, A. (2021). Infección grave por estreptococo del grupo B en la Unidad de Cuidados Intensivos Pediátricos del Centro Hospitalario Pereira Rossell entre los años 2007 y 2017. Archivos de Pediatría del Uruguay, 92(2).

Sampaio, V. R. E. (2017). Sepse: sinais, sintomas e cuidados de enfermagem. Tese de Doutorado. Universidade De Franca.

Shabaan, A. E., Elbaz, L. M., El-Emshaty, W. M. \& Shouman, B. (2018). Role of serum (1, 3)- $\beta$-d-glucan assay in early diagnosis of invasive fungal infections in a neonatal intensive care unit次. Jornal de pediatria, 94, 559-565.

Taniguchi, L. U., Azeveo, L. C. P., Bozza, F. A., Cavalcanti, A. B., Ferreira, E. M., Carrara, F. S. A., Sousa, J. L., Salomão, R. \& Machado, F. R. (2019). Disponibilidade de recursos para tratamento da sepse no Brasil: uma amostra aleatória de instituições brasileiras. Revista Brasileira de Terapia Intensiva, 31, 193-201.

Teixeira, C., Rosa, R. G. \& Friedman, G. (2021) Sepse após a alta da UTI: um problema de saúde pública. Clinical \& Biomedical Research, v. 41 , n. 1.

Westphal, G. A., Pereira, A. B., Fachin, S. M., Barreto, A. C. C., Bornschein, A. C. G. J., Filho, M. C. \& Koenig, A. (2019) Características e desfechos de pacientes com sepse adquirida na comunidade e no hospital. Revista Brasileira de Terapia Intensiva, 31, 71-78.

Westphal, G. A., Pereira, A. B., Fachin, S. M., SperottO, G., Gonçalves, M., Albino, L., Bittencourt, R., FranzinI, V. R. \& Koenig, A. (2018). Um sistema eletrônico de alerta ajuda a reduzir o tempo para diagnóstico de sepse. Revista Brasileira de Terapia Intensiva, 30, 414-422. 